Original article

\title{
A multi stage EEG data classification using k-means and feed forward neural network
}

\author{
Annushree Bablani ${ }^{\mathrm{a}}$, Damodar Reddy Edla ${ }^{\mathrm{a}, *}$, Venkatanareshbabu Kuppili ${ }^{\mathrm{a}}$, Dharavath Ramesh ${ }^{\mathrm{b}}$ \\ ${ }^{a}$ Department of Computer Science and Engineering, National Institute of Technology Goa, India \\ ${ }^{\mathrm{b}}$ Department of Computer Science and Engineering Indian Institute of Technology (ISM), Dhanbad, India
}

\section{A R T I C L E I N F O}

\section{Keywords:}

Electroencephalography

Concealed information test

Event-related potential

Wavelet transform

$K$-means clustering

Neural networks

\begin{abstract}
A B S T R A C T
Objective: Electroencephalography based brain-computer interface techniques are widely used these days as they bring human intentions into reality. Various researchers have proposed different approaches to decode EEG signals for applications like spellers, emotion recognition, lie detection, brain games etc. The ability to analyze concealed behavior is very important for legal and security purposes.

Method: This research aims to identify the concealed behavior of an individual. This paper presents a three-stage "Concealed Information Test" using wavelet transform, k-means clustering and multi-layer feed-forward neural network. The test has been developed by analyzing ERP component (P300) of EEG data during a mock crime scene. The wavelet transforms extracts time and frequency information from raw EEG data. K-means clustering clusters the wavelet coefficients into three clusters. As, neural network models the nonlinear time series data viz EEG, hence it has been utilized for classification of clustered data.

Results: A hybrid 3-stage classification approach is proposed by combining the advantages of all above-mentioned approaches. EEG data is recorded for a "Concealed Information Test", for implementing the proposed framework.

Conclusion: The performance of the proposed system is improved from existing approaches, by providing an accuracy of $83.1 \%$.
\end{abstract}

\section{Introduction}

"Brain Computer Interface (BCI)" is an approach which maps the potential generated inside the brain to a device such as a computer, wheelchairs, prosthetics etc. The brain potentials are recorded as invasive and non-invasive. Non-invasive type acquisition is most widely used for research in brain computing. Various brain computing application has been existing; one of them is, lie detection. To identify the lie, certain tests are conducted. "Concealed Information Test (CIT)" ${ }^{1}$ has been widely used to detect deception. Before performing the CIT, a subject has to participate in a mock crime scene, where he is being trained as a criminal or as an innocent subject. The subject who has been trained as criminal has to go through some crime-related tasks. During the CIT an individual is asked certain questions related to crime scenario and he/she has to reply correctly for these questions. In past, the Polygraphy tests used to be conducted, where if the subject lies, it will be analyzed by their autonomic nervous activity like sweating, mumbling, high heart rate etc. These polygraph tests fail to record if the guilty perform covert activities. Hence, it is not enough to identify the guilty only through autonomic nervous activity. Brain Computer Interface (BCI) provides a solution for this by recording brain activities of guilty through various techniques. One of the most commonly used BCI technique is Electroencephalography (EEG). EEG records brain activity from the brain scalp non-invasively. It is to be noted that, for different brain responses unique EEG signals are generated. These EEG signals are recorded using an acquisition device and are further analyzed. When any cognitive activity is performed, the neural responses elicited are known as Event-Related Potential (ERP). For lie identification when a CIT is performed a certain set of questions are asked to the subject and the cognitive activity is recorded using EEG device. While asking a crime-related question (occurs rarely), a positive peak after $300 \mathrm{~ms}$ of asking the question is elicited. This positive peak is an ERP component and is commonly referred to as P300. These rarely occur, crime-related questions behave as the stimuli for the subject. Hence, these brain neural activities are not controlled with any human intentions and can provide a better way to distinguish between guilty and innocent.

\footnotetext{
* Corresponding author.

E-mail addresses: annubablani@nitgoa.ac.in (A. Bablani), dr.reddy@nitgoa.ac.in (D.R. Edla), venkatanaresh@nitgoa.ac.in (V. Kuppili), ramesh.d.in@ieee.org (D. Ramesh).
} 
Various researchers have applied different approaches to classify the EEG signals generated from the subjects' brain. For performing a CIT, three types of stimuli are usually presented to the subject namely Target, Probe, and Irrelevant. Target stimuli are the set of questions whose answer is already known to both innocent and guilty subjects. Irrelevant are stimuli which are not known to any of the subjects. The probe is the rarely occurring crime-related stimuli. Using these set of stimuli various authors have proposed several different CITs and different approaches for EEG data classification. Farwell et al. ${ }^{2}$ have developed a CIT and analyzed EEG by applying bootstrapping technique on amplitude difference and correlation. Bootstrapping provides the estimate of distribution by randomly sampling when few samples are available. Other works using bootstrapping analysis with a different type of CITs are performed by authors like ${ }^{3},{ }^{4,5}$ and. ${ }^{6}$

EEG data is biological data, hence it consists of a lot of artifacts. For deceit identification P300 component of ERP is used, which cannot be separated in a single trial as the time course of EEG generally overlaps. ${ }^{7}$ To separate P300 and non P300 data, artifact removal using various pattern recognition approaches are applied. Gao et al. ${ }^{8}$ have used the template matching method which is based on "Independent Component Analysis (ICA)". This method has been applied for denoising ERP data and decomposing it into P300 and non-P300 components. Arasteh et al. ${ }^{9}$ applied "Empirical Mode Decomposition (EMD)" as feature extraction approach on raw EEG data. EMD provides a feature of both time and frequency domain by decomposing signal into various Intrinsic Mode Functions (IMFs). A genetic SVM ${ }^{10}$ have been performed through a strict validation structure to prevent from the optimistic result. As a result, various optimal solutions have been obtained, and to find one optimal solution authors have used the most frequent result to reach an optimal solution. In another approach, sparsity-based classification models have been applied to CIT data. ${ }^{11}$ The performance of discriminating property of sparse model has been improved by developing a single framework for reconstruction error and sparse code features. Instead of analyzing all subject's data together, subject-based classification has been performed and received better performance for individual analysis. In this paper, classification of EEG data collected using a concealed information test has been performed. Data has been collected using EEG by performing mock crime scenario involving 10 participants. The EEG signals recorded cannot be analyzed in the raw form, hence it is transformed into meaningful coefficients using wavelets. Wavelet transform provides frequency coefficients of signal with respect to the signal position. It has been successfully used in literature for analysis of signals. The EEG data recorded for this work is unlabeled hence, there is a need to find an approach which provides labelling and classification and in turn improving the performance of the CIT system. This paper presents a hybrid of unsupervised and supervised approach to classify the EEG signals into guilty and innocent. For the same, $k$-means clustering is performed to cluster the EEG data. Using the Silhouette value of the cluster outliers are removed. The clustered data is then given to a multi-layer neural network for classification. The paper is organized into different sections. Section 1 gives the introduction of the problem and discusses existing work, section 2 explains about the proposed three-stage classification, section 3 presents the CIT experimental analysis/results and section 4 concludes the paper followed by the references.

\section{Proposed approach}

Fig. 1 shows the flow of the proposed approach. The proposed approach is achieved in three different stages. At the first stage feature extraction of EEG data is carried out using the wavelet transform approach. Wavelet transform is performed for four levels and the output vector having approximate and detail coefficients are given as input to the clustering algorithm. For clustering, $k$-means clustering is applied for $k=3$. Three clusters are formed, where the first and the second clusters are related to innocent and guilty cases respectively and the third one is related to the noise or artifacts generated during data recording. Using these clusters and their labels, data is fed to a multilayer neural network using the gradient descent algorithm. MLNN performs the classification for deceit identification. In this paper, we have considered three-class classification: innocent, guilty and noise.

\subsection{Three-stage classification}

\subsubsection{Wavelet transform}

For signal transformation approaches like short Fourier transform which transform signal by applying a windowed function centered at zero. The coefficients generated after transformation gives the frequency information of signal at time $t=0$. The coefficients are calculated by the inner products of the signal, let say $x$ with a discrete lattice of coherent states. ${ }^{12}$ The translation using wavelet transform is similar as they also use a function to develop frequency coefficients but with position. Wavelet Transform (WT) ${ }^{12}$ has been widely used as a signal analysis approach. Unlike FFT, WT provides both time and frequency content of the signal. It expresses a signal in the form of a linear combination of a function. It can be expressed as:

$g(x)=|a|^{-\frac{1}{2}} g\left(\frac{x-b}{a}\right)$

Here $g(x)$ is a square integral function tends to infinity. The parameters a, $\mathrm{b}$ gives the frequency component and position component of the wavelet function. If $|a|<<1$, then the function is concentrated towards high frequency range. If $|a|>>1$, then the function is spread and have more low frequency. Hence, wavelet gives better result in the situation when time component is required at high frequencies than at low frequencies. The wavelet functions are obtained by either shifting or scaling a single function known as mother wavelet. After applying the wavelet transform, signals are reduced into different frequency ranges. These frequency ranges are classified into approximation and detail levels.

EEG is a non-stationary signal whose frequency changes with time. To analyze the EEG efficiently, frequency and time domain knowledge is required. ${ }^{13}$ For lie detection application, frequency changes when a subject responds to a particular stimulus at a particular time (latency of signal). Hence, in this paper, we have applied the Discrete Wavelet Transform (DWT) on ERP responses generated during recording. Wavelet function 'db4' (Daubechies wavelet 4) has been applied in this paper as its properties resemble ERP responses. ${ }^{10}$

Multilevel one-dimensional wavelet decomposition has been performed and at each level, decomposition is characterized by a low pass filter and high pass filter operator. Low pass filter gives approximation coefficients whereas high pass filter gives detail coefficients. For feature extraction using wavelet, each trial of the subject has been decomposed into four levels. Every subject has 16 channel data and for each channels' approximate coefficients and detail, coefficients are produced. This is performed for four levels and the process is repeated for 60 trials for all the subjects' EEG data.

\subsection{2. $k$-means clustering}

$k$-means ${ }^{14}, 15$ is the simplest unsupervised clustering algorithm which aims to partition an observation into some cluster based on the mean of the observation. Generally, $k$-means searches for the $k$-partition calculating within cluster sum of square by moving from one cluster to another. For each point let say $i$, it first finds the closest and second closest cluster center $c_{1}$ and $c_{2}$ respectively and assigns the point $i$, with cluster $c_{1}$. The cluster center is then update with the mean of the points in that cluster. In this paper, $k$-means is performed into two steps. Initially, cluster centroid is chosen randomly and the distance of each data point from the centroid is calculated using Euclidean distance using Equation 1

$D(x, c)=\sqrt{\Sigma n}(x-c) 2$

Next, the new cluster centroid is calculated. These two steps are continued until no new centroid are produced. The $k$-means clustering 


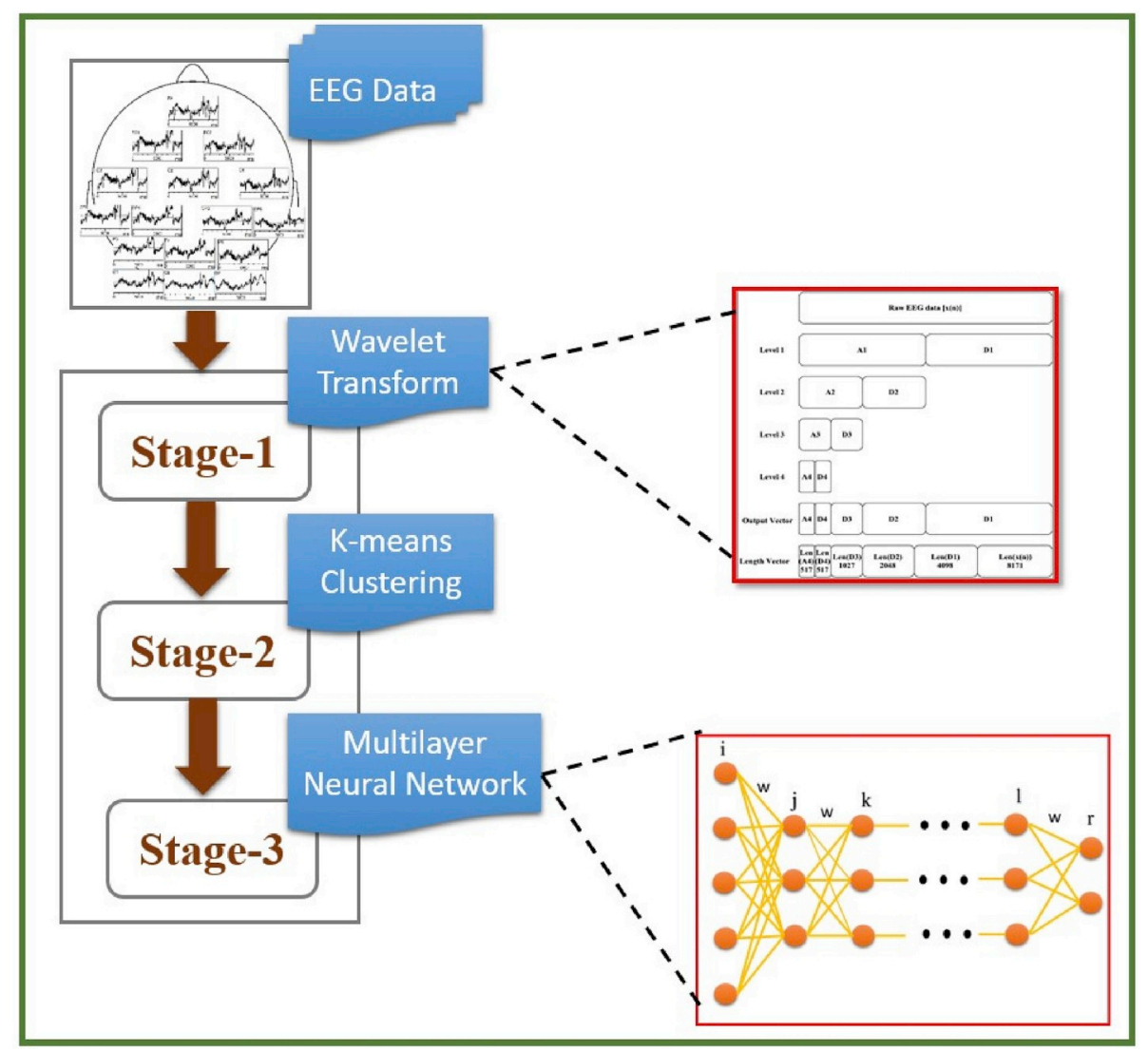

Fig. 1. Proposed three-stage concealed information test.

performance is based on the selection of the cluster. If a large number of clusters are selected it may cause degradation in performance. If fewer clusters are selected it may not provide better separation. The silhouette function ${ }^{16}$ gives the measure of separation of the cluster (according to Equation (2)).

$s(n)=\frac{y(n)-x(n)}{\max [x(n), y(n)]}$

It calculates the average dissimilarity $x(n)$ of the $n$th data point within the same cluster. It interprets that how well the $n$th data point is similar to the clusters. $y(n)$ determines the average dissimilarity of the $n$th data point with the data point of another cluster. From equation (2), it is interpreted that if $s(n)$ is near to 1 (i.e. $x(n) \ll y(n)$ ), then the data point is similar to the cluster. A large value of $y(n)$ indicates more dissimilarity with the neighbouring cluster. Hence, the value of $s(n)$ is near to 1 , then it indicates that data points are properly clustered. Silhouette value gives an interpretation of existing consistency between the clusters. It shows the similarity of an object with its cluster and difference with the other clusters. Fig. 2 shows the silhouette values of the clusters for EEG dataset. The $x$-axis of the figure represent the Silhouette value which lies in the range of $[-1,1]$, and the $y$-axis gives the number of clusters.

\subsubsection{Multi-layer feed forward neural network}

Artificial Neural Network (ANN) ${ }^{17}$ are inspired by the biological neural system having features like parallel computing, nonlinearity, adaptability, responsiveness, and fault tolerance etc. The inputs in neural networks are called neurons, which are connected with weights (can be positive or negative). The inputs with weights are processed through processing units. The processing units consists of a summation part which is finally connected to the output. Inspired by the human nervous system, they are so intelligent that they learn from the output generated at one node and perform further calculations. Similar to a biological neural network, ANN has neurons which are interlinked to each other in the form of layers. The first layer is called an input layer which takes the data and passes it to the next layer through a weighted connection. The output generated by a neuron is given in Equation (3).

$f(x)=\sum_{i=1}^{n} x_{i} w_{i}+$ bias

Where $x_{i}$ is the input neuron, $w_{i}$ is the weight propagated from one layer to another. When the output of a layer is combined to form another layer and so on until the problem is properly classified. This architecture is known as Multi-layer neural network. ${ }^{18}{ }^{19}$ The layers which are added into the network are called Hidden layers. The output at each hidden layer is computed using Equation (3) and is propagated in the whole network in the forward direction. At the output layer, neurons perform a function which is known as activation function or transfer function. To achieve convergence, the neural network learns and propagates the error produced at the output layer back to the input layer. This learning law is called as generalized delta rule which is based on the principle of gradient descent method.

\subsection{Data acquisition}

EEG based CIT has been conducted for 10 participants in two sessions. Among these 10 subjects who participated in the study, nine are male and one is female. Participants have an age between 25 and 30 years. There is no medical record of any psychological disorder found with the participant and they are having a normal or corrected vision. As mentioned earlier in section 1, EEG recording is performed non-invasively, hence to record brain activity EEG electrodes are kept above the brain scalp without injecting them into grey matter. These electrodes are placed using $\mathrm{Ag} / \mathrm{AgCl}$ electrolytic gel at different sites as shown in Fig. 3.

Electrode placement protocol is similar as in Ref. ${ }^{8}$ As a reference, an electrode has been placed on mastoid and another on the forehead as 


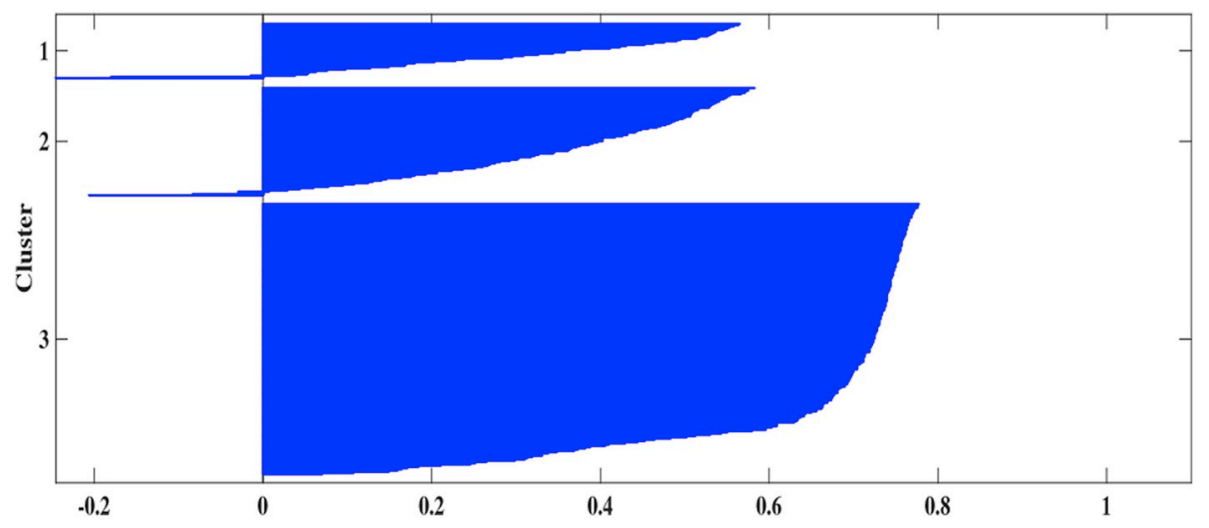

Fig. 2. Silhouette values with three clusters for the concealed information test.

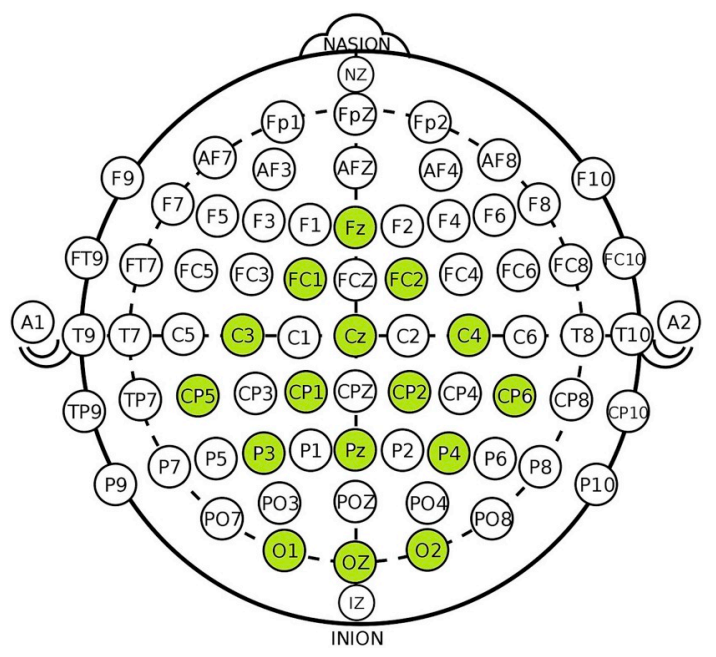

Fig. 3. EEG electrode placement locations.

ground. For signal acquisition, EasyCap ${ }^{20}$ (a 32-Channel EEG Standard Cap Set (Munich, Germany)), a V-amp amplifier, set of 16 electrodes and brain vision recorder ${ }^{21}$ has been used. During the experiment, both the group of subjects are presented a certain set of stimuli, which will generate different ERP responses in subjects' brain. Following the same procedure which was performed in previous studies, ${ }^{2},{ }^{22}$ in this work also, three types of stimuli are presented to the subject i.e. target, irrelevant and probe. Pictorial representation is easy to understand and remembered, hence, instead of asking crime-related questions, we prepared the experiment with the images of crime-related people or incidences. The probe is the image of the person whom guilty subject knows well and is asked to imagine that he/she has committed a crime with (or image of the victim). The experiment begins with presenting the stimuli images to the subject. The subject has to recognize those images and has to answer as "yes" or "no". The images are presented to the subject on a 15.4-inch display screen. Each image has been displayed for $1.1 \mathrm{~s}$ followed by a blank image for $2 \mathrm{~s}$. So, a total of ten images are presented to the subject, out of ten images, seven are irrelevant images, two are target images and one is probe image. Fig. 4 depicts the timeline followed during the experiment. The experiment goes into two sessions, where each session have been conducted for 30 trials for a single subject, so a total of 600 trials $(2 \times 30 \times 1 \times 10)$ have been conducted during experimental sessions. So, for one subject one session has been performed in one sitting i.e. 30 trial for $31 \mathrm{~s}$ which will be approximately $930 \mathrm{~s}$ (or $15.5 \mathrm{~min}$ ). After the first session is over, the subject is given a break for $15 \mathrm{~min}$ and then the next session is started. During recording, it has been kept in mind that the subject should not feel headache or uneasy. If any such case occurs, the experiment is halted until the subject is not ready to participate in the experiment.

During the guilty session, subjects are instructed to respond "yes" for the target image, whereas "no" for probe image and irrelevant image. During the innocent session, subjects are instructed to respond "yes" target image, whereas "no" for probe image and irrelevant image. EEG signals have been recorded in a real environment, hence consist lot of artifacts. To remove the high-frequency signal, the bandpass filter has been applied. Bandpass filter removes a given band of frequency from the signal without degrading signal quality. In this study, the bandpass filter is applied in a range of $0.3 \mathrm{~Hz}-30 \mathrm{~Hz}$. This is the range of frequency usually analyzed when the mental task is performed by the subject. $^{3}$

\section{Experimental results and analysis}

A CIT has been performed to analyze human behavior whilst lying. The EEG data is converted into numerical attributes using Brain Vision Analyzer 2.1. ${ }^{21}$ Experiment is conducted using Intel(R) Core(TM) i7$4790 \mathrm{CPU} @ 3.60 \mathrm{GHz}$, with 8 GB RAM. Furthermore, the implementation is performed using MATLAB 2016a. The amplitude values recorded from the scalp during the guilty session and innocent sessions are shown in Fig. 5a and Fig. 5b respectively. The difference between the signals generated from innocent and guilty subjects using their power spectra have been plotted. The power spectra of subject 4 have been plotted as shown in Fig. 6 a for the guilty session and Fig. $6 \mathrm{~b}$ for the innocent session.

From Fig. 6a, the first scalp (from left) represents the distribution of power during the guilty session at $6 \mathrm{~Hz}$, where, it is concentrated at the frontal and parietal lobe. The other two scalp maps give the power

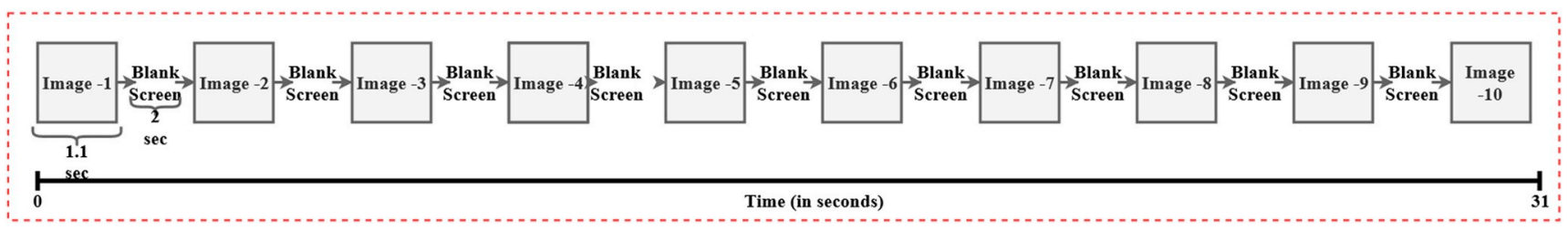

Fig. 4. Timeline of the experiment. 


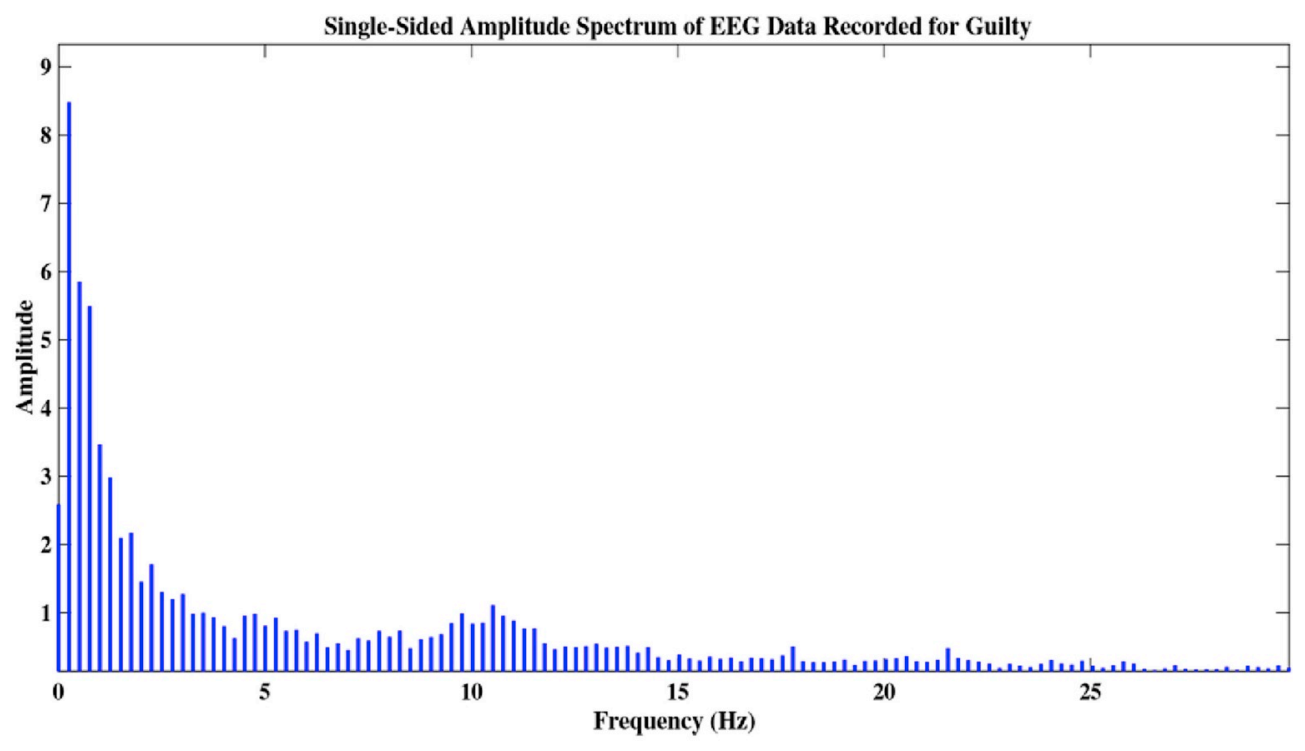

(a) Guilty session

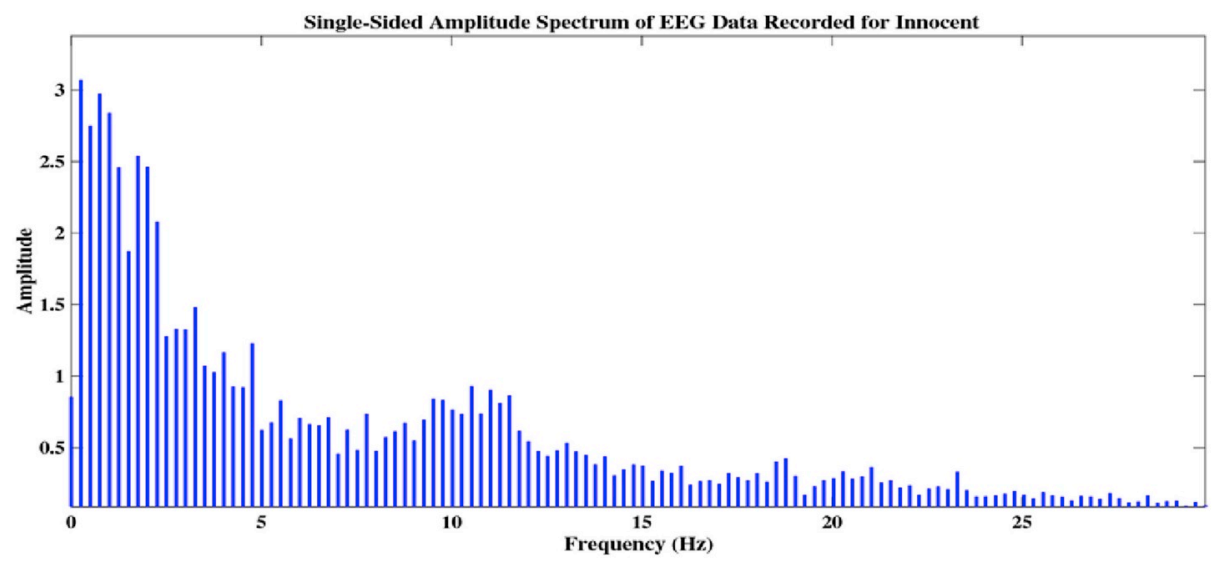

(b) Innocent session

Fig. 5. Amplitude spectrum of EEG data during two sessions.

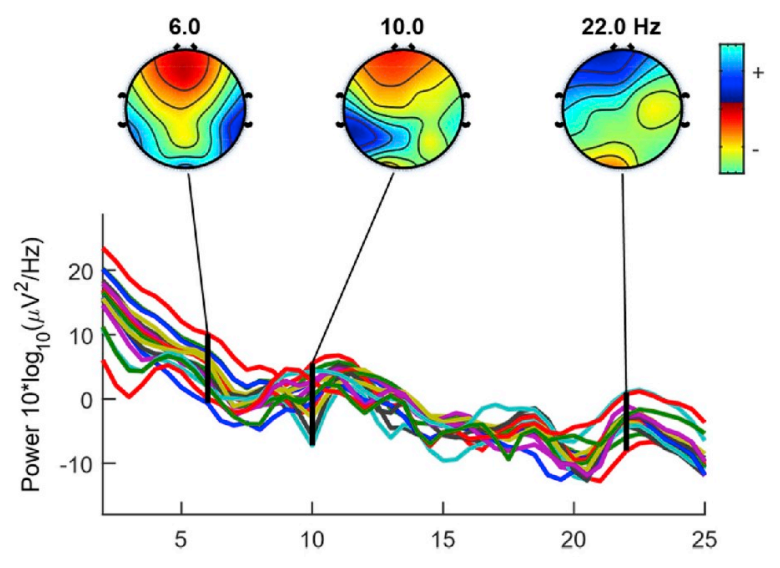

(a) Guilty session

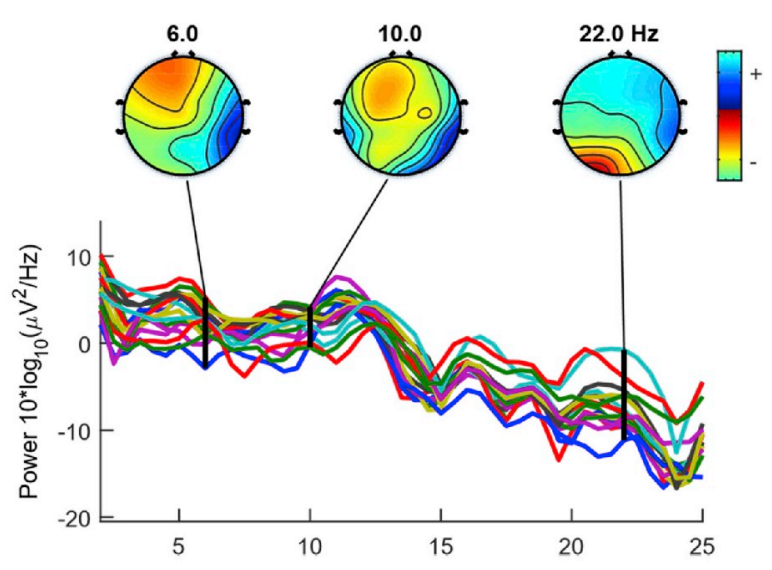

(b) Innocent session

Fig. 6. Channel spectra of EEG data during two sessions. 


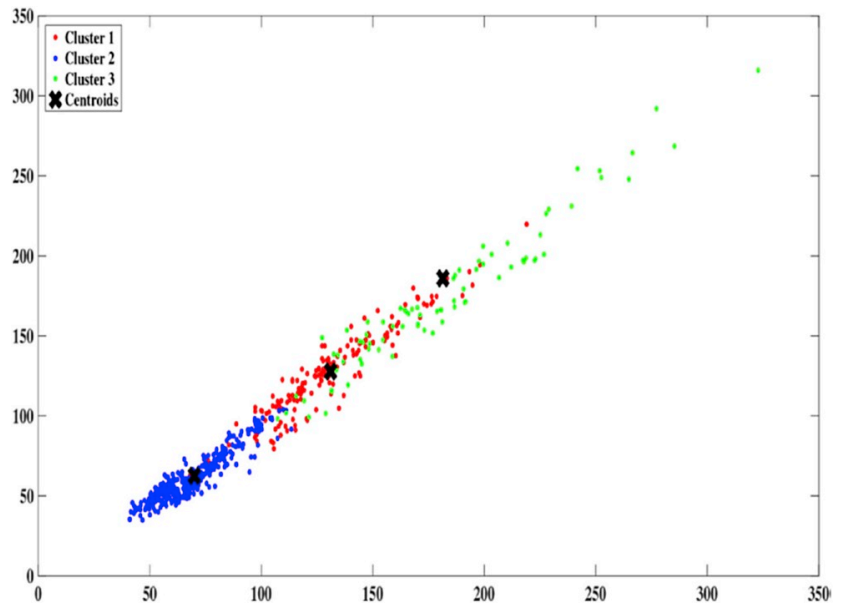

Fig. 7. Clustering using K-means.

distribution at $10 \mathrm{~Hz}$ and $22 \mathrm{~Hz}$. Similarly, distribution for the innocent session is shown in Fig. 6b. The $\mathrm{x}$-axis for Figs. 5 and 6 represents the frequency of EEG signal plotted against amplitude and power respectively.

For the analysis of data, the wavelet transform has been performed on single subject single-trial EEG data. After wavelet transform, the data of 10 subjects are combined in a matrix having dimension number of trials $\times$ number of wavelet coefficients. This data matrix is clustered using $k$-means clustering algorithm for $k=3$ clusters (Fig. 7).

After clustering the data, outliers are removed and clusters with their labels are passed through the neural network. At this stage, the classification of data in a single iteration cannot give accurate the results, hence, 10 fold cross-validation has been applied to classify EEG data. Numerical EEG data is randomly partitioned into training and testing tests via 10 fold cross-validation. Data is divided into 10 parts, where 9 parts will be trained and one will be tested. 10 fold crossvalidation is applied as it improves the performance and reliability of the system. For calculating the classification performance, various performance measures like accuracy, sensitivity ${ }^{23}$ and F1-score ${ }^{24}$ are utilized which are given in equations (4)-(6).

Accuracy $=\frac{G G+I I}{G G+I I+I G+G I}$

Sensitivity $=\frac{G G}{G G+G I}$

F1score $=\frac{2 G G}{2 G G+I I+I G}$

Where GG represents the guilty class which is classified as guilty by the classifier, II represents the innocent class which is observed as innocent by the classifier, IG represents the innocent class which is categorized as guilty by the classifier and GI represents the guilty class which is classified as innocent by the classifier.

To validate the proposed approach, it has been compared with different approaches. The existing approaches are applied to EEG data recorded for this work. From the results, it is envisaged that the proposed hybrid approach gives better results than existing supervised approaches. The results have been evaluated by applying recorded data on existing methods. InRef. 25, the author has applied non-parametric LDA as feature extraction approach and later constructed a hierarchical feature space which has been classified using KNN. They have used only one electrode EEG data for classification, which have pros and cons both. One electrode is easy to use and doesn't provide the subject with any kind of discomfort. But from the accuracy point of view, one electrode data is not sufficient to analyze the behavior of a subject. InRef. 9 authors have applied EMD as feature extraction approach
Table 1

Results of the proposed approach and comparison with some existing works.

\begin{tabular}{|c|c|c|c|c|}
\hline Classifier & Accuracy & Sensitivity & Specificity & F1-score \\
\hline $\begin{array}{c}\text { Non-parametric LDA } \\
+ \text { KNN [25] }\end{array}$ & $76.8 \%$ & $70.0 \%$ & $73.1 \%$ & - \\
\hline EMD + LDA [9 ] & $80.0 \%$ & $75.7 \%$ & $75.7 \%$ & - \\
\hline ICA + SVM [8] & $60.17 \%$ & $51.33 \%$ & $67.83 \%$ & - \\
\hline Proposed & $83.1 \%$ & $95 \%$ & $89.5 \%$ & - \\
\hline
\end{tabular}

which works as good as a wavelet. Further LDA as classification approach is utilized. InRef. 8, authors have used ICA and SVM for classification of P300 data collected for CIT. For the same, authors have separated non-P300 and P300 data using ICA and then applied SVM classifier. Table 1 tabulates and compares the result achieved using the proposed approach with existing methods. The proposed approach provides an accuracy of $83.1 \%$ and giving $95 \%$ sensitivity. It is inferred that the proposed approach classifies true class almost correctly. As a performance measure, the F1 score is also calculated. Using the proposed approach $89.5 \%$ of F1-score value has been achieved.

\section{Conclusion}

The brain-computer interface is the topic of interest for researchers these days. One of the EEG based BCI application is the Concealed Information Test. In this paper, a three-state Concealed Information Test is proposed. In which, the first stage consists of extracting the time and frequency features from EEG data using wavelet transform. The second stage utilizes the $k$-means clustering approach to cluster the wavelet coefficients. The final stage classifies the clustered data using Multilayer Feed Forward Neural Network. An EEG device has been used to acquire the data on which the proposed method is applied. The approach has been compared with other existing approaches based on accuracy and sensitivity. It has achieved an improved performance when compared to existing approach with $83.1 \%$ accuracy and $95 \%$ sensitivity.

\section{Funding}

There is no funding source.

\section{Declaration of competing interest}

The authors declare that there is no conflict of interest regarding this paper.

\section{References}

1. Lykken DT. The GSR in the detection of guilt. J Appl Psychol. 1959;43(6):385

2. Farwell LA, Donchin E. The truth will out: interrogative polygraphy (lie detection) with event-related brain potentials. Psychophysiology. 1991;28(5):531-547.

3. Rosenfeld JP, Soskins M, Bosh G, et al. Simple, effective countermeasures to p300based tests of detection of concealed information. Psychophysiology. 2004;41(2):205-219.

4. Rosenfeld JP, Labkovsky E, Winograd M, et al. The complex trial protocol (ctp): a new, countermeasure-resistant, accurate, p300-based method for detection of concealed information. Psychophysiology. 2008;45(6):906-919.

5. Kubo K, Nittono H. The role of intention to conceal in the p300-based concealed information test. Appl Psychophysiol Biofeedback. 2009;34(3):227-235.

6. Meixner JB, Rosenfeld JP. A mock terrorism application of the p300-based concealed information test. Psychophysiology. 2011;48(2):149-154.

7. Jung TP, Makeig S, Humphries C, et al. Removing electroencephalographic artifacts by blind source separation. Psychophysiology. 2000;37(2):163-178.

8. Gao J, Lu L, Yang Y, et al. A novel concealed information test method based on independent component analysis and support vector machine. Clin EEG Neurosci. 2012;43(1):54-63.

9. Arasteh A, Moradi MH, Janghorbani A. A novel method based on empirical mode decomposition for p300-based detection of deception. IEEE Trans Inf Forensics Secur. 2016;11(11):2584-2593.

10. Farahani ED, Moradi MH. Multimodal detection of concealed information using geneticsvm classifier with strict validation structure. Inf Med Unlocked. 2017. 
11. Akhavan A, Moradi MH, Vand SR. Subject-based discriminative sparse representation model for detection of concealed information. Comput Methods Progr Biomed. 2017;143:25-33.

12. Daubechies I. The wavelet transforms time-frequency localization and signals analysis. IEEE Trans Inf Theory. 1990;36(5):961-1005.

13. Jenke R, Peer A, Buss M. Feature extraction and selection for emotion recognition from EEG. IEEE Trans Affect Comput. 2014;5(3):327-339.

14. MacQueen J, et al. Some methods for classification and analysis of multivariate observations. Proceedings of the Fifth Berkeley Symposium on Mathematical Statistics and Probability. vol 1. 1967; 1967:281-297 Oakland, CA, USA.

15. Benmouiza K, Cheknane A. Forecasting hourly global solar radiation using hybrid kmeans and nonlinear autoregressive neural network models. Energy Convers Manag. 2013;75:561-569.

16. Rousseeuw PJ. Silhouettes: a graphical aid to the interpretation and validation of cluster analysis. J Comput Appl Math. 1987;20:53-65.

17. McCulloch WS, Pitts W. A logical calculus of the ideas immanent in nervous activity. Bull Math Biophys. 1943;5(4):115-133 Dec.
18. Hornik K, Stinchcombe M, White H. Multilayer feedforward networks are universal approximators. Neural Netw. 1989;2(5):359-366

19. Svozil D, Kvasnicka V, Pospichal J. Introduction to multi-layer feed-forward neural networks. Chemometr Intell Lab Syst. 1997;39(1):43-62.

20. Easycap. 2017; 2017http://www.easycap.de/e/products/products.htm15.

21. Brain Products. 2017; 2017http://www.brainproducts.com/.

22. Abootalebi V, Moradi MH, Khalilzadeh MA. A new approach for EEG feature extraction in p300-based lie detection. Comput Methods Progr Biomed. 2009;94(1):48-57.

23. Zhu W, Zeng N, Wang N, et al. Sensitivity, specificity, accuracy, associated confidence interval and roc analysis with practical SAS implementations. NESUG Proceedings: Health Care and Life Sciences, Baltimore, Maryland. 2010; 2010:67.

24. Powers DM. Evaluation: From Precision, Recall and F-Measure to Roc, Informedness, Markedness and Correlation. 2011; 2011.

25. Wang D, Miao D, Blohm G. A new method for EEG-based concealed information test. IEEE Trans Inf Forensics Secur. 2013;8(3):520-527. 